\title{
A NEW PICARD TYPE THEOREM CONCERNING ELLIPTIC FUNCTIONS
}

\author{
Qiaoyu Chen, Xuecheng Pang and Pai Yang \\ Shanghai Lixin University of Commerce, School of Mathematics and Information Science \\ Shanghai 201620, P. R. China; goodluckqiaoyu@126.com \\ East China Normal University, Department of Mathematics \\ Shanghai 200241, P. R. China; xcpang@math.ecnu.edu.cn \\ Chengdu University of Information Technology, College of Applied Mathematics \\ Chengdu 610225, P. R. China; yangpai@cuit.edu.cn
}

\begin{abstract}
Let $k \geq 2$ be an integer, let $h$ be a nonconstant elliptic function, and let $f$ be a nonconstant meromorphic function in $\mathbf{C}$, all of whose zeros have multiplicity at least $k+1$, except possibly finitely many. If $T(r, h)=o\{T(r, f)\}$ as $r \rightarrow \infty$, then $f^{(k)}=h$ has infinitely many solutions (including the possibility of infinitely many common poles of $f$ and $h$ ).
\end{abstract}

\section{Introduction and our result}

Notation. Throughout $\mathbf{C}$ is the complex plane and $D$ is a domain in $\mathbf{C}$. For $z_{0} \in \mathbf{C}, r>0, \Delta\left(z_{0}, r\right)=\left\{z|| z-z_{0} \mid<r\right\}, \Delta^{\prime}\left(z_{0}, r\right)=\left\{z|0<| z-z_{0} \mid<r\right\}$ and $\Delta=\Delta(0,1)$. Let $n(r, f)$ denote the number of poles of $f(z)$ in $\Delta(0, r)$ (counting multiplicity) and let $n\left(\Delta\left(z_{0}, r\right), \frac{1}{f}\right)$ denote the number of zeros of $f(z)$ in $\Delta\left(z_{0}, r\right)$ (counting multiplicity). We write $f_{n} \stackrel{\chi}{\Longrightarrow} f$ in $D$ to indicate that the sequence $\left\{f_{n}\right\}$ converges to $f$ in the spherical metric uniformly on compact subsets of $D$ and $f_{n} \Rightarrow f$ in $D$ if the convergence is in the Euclidean metric.

Let $f$ be a meromorphic function,

$$
f^{\#}(z)=\frac{\left|f^{\prime}(z)\right|}{1+|f(z)|^{2}}, \quad S(D, f)=\frac{1}{\pi} \iint_{D}\left[f^{\#}(z)\right]^{2} \mathrm{~d} x \mathrm{~d} y \text { and } S(r, f)=S(\Delta(0, r), f) .
$$

The Ahlfors-Shimizu characteristic is defined by

$$
T(r, f)=\int_{0}^{r} \frac{S(t, f)}{t} \mathrm{~d} t
$$

Suppose that $h$ is a meromorphic function in $\mathbf{C}$ and there exist two non-zero complex numbers $\omega_{1}$ and $\omega_{2}$ with $\omega_{1} / \omega_{2}$ not real such that $h\left(z+\omega_{1}\right)=h\left(z+\omega_{2}\right)=h(z)$ for all $z$ in C. $h$ is called to be an elliptic function [1].

In 1959, Hayman proved the following seminal result, which has come to be known as Hayman's alternative.

Theorem A. [5, Theorem 1] Let $f$ be a transcendental meromorphic function in $\mathbf{C}, a \in \mathbf{C}, b \in \mathbf{C} \backslash\{0\}, k \in Z^{+}$. Then

doi:10.5186/aasfm.2015.4001

2010 Mathematics Subject Classification: Primary 30D35, 30D45.

Key words: Meromorphic function, elliptic function, normal family.

This work was supported by the National Natural Science Foundation of China (Grant Nos. 11371139; 11001081) and the Scientific Research Foundation of CUIT (Grant No. KYTZ201403). 
(i) $f$ assumes each value $a$ infinitely often, or

(ii) $f^{(k)}$ assumes each value $b$ infinitely often for $k=1,2, \cdots$.

Recently, researchers replaced the condition $f \neq 0$ which is related to $a=0$ in Theorem A with the assumption that all zeros of $f$ have sufficiently high multiplicity.

Theorem B. [8, Theorem 3] Let $f$ be a transcendental meromorphic function in $\mathbf{C}$, all of whose zeros have multiplicity at least 3 . Then $f^{\prime}$ assumes each nonzero complex value infinitely often.

In 2006, Nevo, Pang and Zalcman promoted Theorem B and obtained the following result.

Theorem C. [7, Theorem 1] Let $f$ be a transcendental meromorphic function in $\mathbf{C}$, all but finitely many of whose zeros are multiple, and let $R \neq \equiv 0$ be a rational function. Then $f^{\prime}-R$ has infinitely many zeros.

In 2013, Yang and Nevo proved the following result.

Theorem D. [11, Theorem] Let $f$ be a nonconstant meromorphic function in $\mathbf{C}$ and $h$ be a nonconstant elliptic function. If all zeros of $f$ are multiple except finitely many and $T(r, h)=o\{T(r, f)\}$ as $r \rightarrow \infty$, then $f^{\prime}=h$ has infinitely many solutions (including the possibility of infinitely many common poles of $f$ and $h$ ).

Naturally, we ask a question: In Theorem D, whether $f^{(k)}, k(\geq 2) \in Z^{+}$, has the similar property? In this paper, we give a positive answer.

Theorem 1.1. Let $k \geq 2$ be an integer, let $h$ be a nonconstant elliptic function, and let $f$ be a nonconstant meromorphic function in $\mathbf{C}$, all of whose zeros have multiplicity at least $k+1$, except possibly finitely many. If $T(r, h)=o\{T(r, f)\}$ as $r \rightarrow \infty$, then $f^{(k)}=h$ has infinitely many solutions (including the possibility of infinitely many common poles of $f$ and $h$ ).

\section{Preliminary results}

In order to prove the Theorem, we need the following lemmas.

Lemma 2.1. [2, Corollary 2] If $h(z)$ is a nonconstant elliptic function with primitive periods $\omega_{1}, \omega_{2}$, where $\omega_{1} / \omega_{2}$ is not real, then $T(r, h)=A r^{2}(1+o(1))$ as $r \rightarrow \infty$, where $A>0$ is a constant.

Lemma 2.2. [11, Lemma 3.6] Let $\left\{f_{n}\right\}$ be a sequence of meromorphic functions in $\Delta\left(z_{0}, r\right)$. Suppose that

(a) there exists $M_{1}>0$ such that for each $n \in \mathbf{N}, n\left(\Delta\left(z_{0}, r\right), \frac{1}{f_{n}}\right)<M_{1}$,

(b) $f_{n} \stackrel{\chi}{\Longrightarrow} f$, in $\Delta^{\prime}\left(z_{0}, r\right)$, where $f$ is a nonconstant meromorphic function or $f \equiv \infty$ in $\Delta^{\prime}\left(z_{0}, r\right)$.

Then there exists $M_{2}>0$ such that, for sufficiently large $n$,

$$
S\left(\Delta\left(z_{0}, \frac{r}{2}\right), f_{n}\right)<M_{2}
$$

Lemma 2.3. [9, Lemma 6] Let $k, l$ be positive integers, and let $R(z)$ be a rational function, all of whose zeros have multiplicity at least $k$, satisfying $R^{(k)}(z) \neq z^{-l}$ in C. Then $R(z)$ is a constant. 
Lemma 2.4. [3, Lemma 12] Let $R$ be a nonconstant rational function satisfying $R^{\prime} \neq 0$ in $\mathbf{C}$. Then either $R(z)=a z+b$ or $R(z)=\frac{a}{(z+c)^{n}}+b$, where $n \in \mathbf{N}$ and $a(\neq 0), b, c \in \mathbf{C}$.

Lemma 2.5. [6, Lemma 3] Let $\left\{f_{n}\right\}$ be a sequence of meromorphic functions in $\Delta\left(z_{0}, r\right)$, and let $\left\{\psi_{n}\right\}$ be a sequence of holomorphic functions in $\Delta\left(z_{0}, r\right)$ such that $\psi_{n} \Rightarrow \psi$, where $\psi(z) \neq 0, \infty$ in $\Delta\left(z_{0}, r\right)$. If $f_{n}(z) \neq 0$ and $f_{n}^{(k)}(z) \neq \psi_{n}(z)$ for all $z$ in $\Delta\left(z_{0}, r\right)$, then $\left\{f_{n}\right\}$ is normal in $\Delta\left(z_{0}, r\right)$.

Using the same proof method in [11, Lemma 3.4, Lemma 3.5], we can get the following two results respectively.

Lemma 2.6. Let $\left\{f_{n}\right\}$ and $\left\{\psi_{n}\right\}$ be two sequences of meromorphic functions in $D$. Let $f(z)$ and $\psi(z)$ be two meromorphic functions in D. Suppose that

(a) $f_{n}(z) \stackrel{\chi}{\Longrightarrow} f(z)$ and $\psi_{n}(z) \stackrel{\chi}{\Longrightarrow} \psi(z)$ in $D$,

(b) $f_{n}^{(k)}(z) \neq \psi_{n}(z)$ in $D$.

Then, either $f^{(k)}(z) \equiv \psi(z)$ or $f^{(k)}(z) \neq \psi(z)$ in $D$.

Proof. Suppose that $f^{(k)}(z) \not \equiv \psi(z)$ in $D$. Set $A=f^{-1}(\infty) \cup \psi^{-1}(\infty) \cup\left(f^{(k)}-\right.$ $\psi)^{-1}(0)$. By (a) and (b), we have

$$
\infty \neq \frac{1}{f_{n}^{(k)}-\psi_{n}} \Longrightarrow \frac{1}{f^{(k)}-\psi} \quad \text { in } D \backslash A .
$$

Since $\frac{1}{f_{n}^{(k)}-\psi_{n}}$ is holomorphic in $D$ and $A$ has no accumulation points in $D$, we have

$$
\frac{1}{f_{n}^{(k)}-\psi_{n}} \Longrightarrow \frac{1}{f^{(k)}-\psi} \text { in } D
$$

Thus, $\frac{1}{f^{(k)}-\psi}$ is a holomorphic function in $D$ and then $f^{(k)}-\psi \neq 0$ in $D$.

In order to show that $f^{(k)} \neq \psi$ in $D$, we need only show that $f$ and $\psi$ have no common poles in $D$. Otherwise, we assume that $z_{0} \in D$ is a pole of order $m_{1}$ of $f$ and a pole of order $m_{2}$ of $\psi$. Let $m=\max \left\{m_{1}+k, m_{2}\right\}$. Obviously, $z_{0}$ is a zero of $\frac{1}{f^{(k)}-\psi}$ of order at most $m$.

By (a) and Hurwitz's Theorem, there exists $\delta^{*}$ such that $\Delta\left(z_{0}, 2 \delta^{*}\right) \subset D$ and for each $\delta \in\left(0, \delta^{*}\right), f_{n}$ and $b_{n}$ have at least $m_{1}$ and $m_{2}$ (counting multiplicities) poles respectively in $\Delta\left(z_{0}, \delta\right)$ for sufficiently large $n$. By (b), $f_{n}$ and $\psi_{n}$ have no common poles in $\Delta\left(z_{0}, \delta\right)$, and hence $f_{n}^{(k)}-\psi_{n}$ has at least $m_{1}+k+m_{2}$ poles (counting multiplicities $)$ in $\Delta\left(z_{0}, \delta\right)$. Since $\delta$ can be made arbitrarily small, $z_{0}$ is a zero of $\frac{1}{f^{(k)}-\psi}$ of order at least $m_{1}+k+m_{2}$ by (2.6.1). Thus, $m_{1}+k+m_{2}>m$. This is a contradiction.

Lemma 2.7. Let $k, l$ be positive integers, and let $\left\{f_{n}\right\}$ be a sequence of meromorphic functions in $\Delta$, all of whose zeros have multiplicity at least $k+1$. Let $\left\{b_{n}\right\}$ be a sequence of holomorphic functions in $\Delta$ such that $b_{n} \Rightarrow 1$, and suppose that

(a) $f_{n}^{(k)}(z) \neq z^{l} b_{n}(z)$ in $\Delta$,

(b) there exist points $z_{n} \rightarrow 0$ such that $f_{n}\left(z_{n}\right)=0$,

(c) $f_{n}(z) \stackrel{\chi}{\Longrightarrow} f(z)$ in $\Delta^{\prime}$, where $f(z)$ is a meromorphic function in $\Delta^{\prime}$. Then $f^{(k)}(z) \equiv z^{l}$ in $\Delta^{\prime}$. 
Proof. Let $F_{n}(z)=\frac{f_{n}^{(k)}(z)}{z^{l}}$. Since $b_{n} \Rightarrow 1$ in $\Delta$ and (a), we have for sufficiently large $n, f_{n}^{(k)}(0) \neq 0$ and hence $F_{n}(0)=\infty$. Without loss of generality, we may assume that for all $n, f_{n}^{(k)}(0) \neq 0$ and $F_{n}(0)=\infty$. Since all zeros of $\left\{f_{n}(z)\right\}$ have multiplicity at least $k+1$, we have $f_{n}(0) \neq 0$. Hence we have $z_{n} \neq 0$ and $F_{n}\left(z_{n}\right)=0$.

We claim that $\left\{F_{n}(z)\right\}$ is not normal at 0 and hence $\left\{\frac{f_{n}^{(k)}(z)}{z^{l} b_{n}(z)}-1\right\}$ is also not normal at 0 . Indeed, since $F_{n}\left(z_{n}\right)=0$ and $F_{n}(0)=\infty$, the family $\left\{F_{n}(\zeta)\right\}$ is not equicontinuous at 0 and hence cannot be normal at 0 .

By (a) and (c), we have

$$
0 \neq \frac{f_{n}^{(k)}(z)}{z^{l} b_{n}(z)}-1 \Longrightarrow \frac{f^{(k)}(z)}{z^{l}}-1, \quad z \in \Delta^{\prime} \backslash f^{-1}(\infty) .
$$

By Hurwitz's Theorem, either $\frac{f^{(k)}(z)}{z^{l}}-1 \equiv 0$ in $\Delta^{\prime}$, or $\frac{f^{(k)}(z)}{z^{l}}-1 \neq 0$ in $\Delta^{\prime}$. Suppose that $\frac{f^{(k)}(z)}{z^{l}}-1 \neq 0$ in $\Delta^{\prime}$. Since $f(z)$ is a meromorphic function, then there exists $\delta>0$ such that $f(z)$ has no poles on $\Gamma(0, \delta)$ and $f_{n}^{(k)}(z)$ converges uniformly to $f^{(k)}(\zeta)$ on $\Gamma(0, \delta)$. Now, we have

$$
\infty \neq \frac{1}{\frac{f_{n}^{(k)}(z)}{z^{l} b_{n}(z)}-1} \Longrightarrow \frac{1}{\frac{f^{(k)}(z)}{z^{l}}-1}, \quad z \in \Gamma(0, \delta) .
$$

Since the function in the left hand side is holomorphic, we have by the maximum principle that this holds throughout $\Delta(0, \delta)$. So $\left\{\frac{f_{n}^{(k)}(z)}{z^{l} b_{n}(z)}-1\right\}$ is normal at 0 . A contradiction. Thus, $\frac{f^{(k)}(z)}{z^{l}}-1 \equiv 0$ in $\Delta^{\prime}$. Obviously, $f^{(k)}(z) \equiv z^{l}$ in $\Delta^{\prime}$.

Lemma 2.8. Let $\left\{a_{k}\right\}$ be a sequence in $D$ which has no accumulation points in $D$. Let $\left\{\psi_{n}\right\}$ be a sequence of holomorphic functions in $D$ such that $\psi_{n} \Rightarrow \psi$ in $D$, where $\psi \neq 0, \infty$ in $D$. Let $\left\{f_{n}\right\}$ be a sequence of meromorphic functions in $D$, all of whose zeros are of multiplicity at least $k+1$. Suppose that

(a) $f_{n}(z) \stackrel{\chi}{\Longrightarrow} f(z)$ in $D \backslash\left\{a_{j}\right\}_{j=1}^{\infty}$,

(b) no subsequence of $\left\{f_{n}\right\}$ is normal at $a_{1}$,

(c) for all $n \in \mathbf{N}, f_{n}^{(k)}(z) \neq \psi_{n}(z)$ in $D$.

Then

(d1) there exists $\delta>0$ such that for all sufficiently large $n, f_{n}$ has a single (multiple) zero in $\Delta\left(a_{1}, \delta\right)$,

(d2) there exists $\eta_{0}>0$ such that for each $0<\eta<\eta_{0}, f_{n}$ has a single simple pole in $\Delta\left(a_{1}, \eta\right)$ for all sufficiently large $n$, and

(e) $f(z)=\int_{a_{1}}^{z} \int_{a_{1}}^{\zeta_{1}} \cdots \int_{a_{1}}^{\zeta_{k-1}} \psi\left(\zeta_{k}\right) \mathrm{d} \zeta_{k} \mathrm{~d} \zeta_{k-1} \cdots \mathrm{d} \zeta_{1}$. Equivalently, $f$ extends to an analytic function in $D \backslash\left\{a_{j}\right\}_{j=2}^{\infty}$ such that $f^{(k)}=\psi(z)$ and $f^{(j)}\left(a_{1}\right)=0, j=$ $0,1,2, \cdots, k-1$.

Remark. Since Lemma 2.8 is not stated explicitly in [6], let us indicate how it follows from the results of that paper. Let us now assume that (d1) has been shown to hold. We obtain (d2) and (e) as well, which follow from [6, Lemma 7]. Now let us show that (d1) must hold. Suppose not. Then it follows from Lemma 2.5 that taking a subsequence and renumbering, we may assume that in any neighborhood of $a_{1}, f_{n}$ 
has at least two zeros for sufficiently large $n$. We derive a contradiction by the same proof method in [6, Theorem 1, Case II, pp. 13-16].

According to the proof method in [6, Theorem 1 and Corollary 1], we get the following result.

Lemma 2.9. Let $k$ be a positive integer and let $\left\{f_{n}\right\}$ be a sequence of meromorphic functions in $D$, all of whose zeros have multiplicity at least $k+1$. Let $\left\{\psi_{n}\right\}$ be a sequence of meromorphic functions in $D$ such that $\psi_{n} \stackrel{\chi}{\Longrightarrow} \psi$, where $\psi(z) \not \equiv 0, \infty$ in $D$. If $f_{n}^{(k)} \neq \psi_{n}$ for all $z$ in $D$, then $\left\{f_{n}\right\}$ is quasinormal in $D$.

By means of the proof method in [12, Theorem $1^{\prime}$, p. 67] and $[9$, Theorem 1], we get the following result clearly.

Lemma 2.10. Let $k \geq 2$ be an integer and let $\left\{f_{n}\right\}$ be a sequence of meromorphic functions in $D$, all of whose poles are multiple and whose zeros all have multiplicity at least $k+1$. Let $\left\{h_{n}\right\}$ be a sequence of meromorphic functions in $D$ such that $h_{n} \stackrel{\chi}{\longrightarrow} h$ in $D$, where $h \not \equiv 0, \infty$. Suppose $h$ and $h_{n}$ have the same zeros and poles all with the same multiplicity and

$$
f_{n}^{(k)}(z) \neq h_{n}(z) \text { for all } z \text { in } D .
$$

Then $\left\{f_{n}\right\}$ is normal in $D$.

In 2013, Yang and Liu promoted Theorem C and obtained the result.

Lemma 2.11. [10, Theorem 1] Let $k$ be a positive integer, let $f$ be a transcendental meromorphic function in $\mathbf{C}$ and let $R \not \equiv 0$ be a rational function. Suppose that all zeros of $f$ have multiplicity at least $k+1$, except possibly finite many. Then $f^{(k)}-R$ has infinitely many zeros.

\section{Auxiliary lemmas}

Lemma 3.1. Let $k, l$ be positive integers and let $R(z)$ be a rational function. Suppose that $R^{(k)}(z) \neq z^{l}$ in $\mathbf{C}$, then

$$
R(z)=\frac{\prod_{i=1}^{l+n+1}\left(z-\gamma_{i}\right)}{(l+k)(l+k-1) \cdots(l+1)(z-\beta)^{n-k+1}}
$$

where $n \geq k$ is an integer and $\gamma_{i}, \beta \in \mathbf{C}, 1 \leq i \leq l+n+1$.

Proof. Obviously, $\left(R^{(k-1)}(z)-\frac{z^{l+1}}{l+1}\right)^{\prime} \neq 0$. Then $R^{(k-1)}(z)-\frac{z^{l+1}}{l+1}$ is a nonconstant rational function. By Lemma 2.4,

$$
R^{(k-1)}(z)=\frac{z^{l+1}}{l+1}+a z+b \quad \text { or } \quad R^{(k-1)}(z)=\frac{z^{l+1}}{l+1}+\frac{a}{(z+c)^{n}}+b .
$$

where $n \geq k$ is an integer and $a(\neq 0), b, c \in \mathbf{C}$. So

or

$$
R(z)=\frac{z^{l+k}}{(l+k) \cdots(l+1)}+P_{k}(z)
$$

$$
R(z)=\frac{z^{l+k}}{(l+k) \cdots(l+1)}+\frac{c_{1}}{(z+c)^{n-k+1}}+P_{k-1}(z)
$$


where $P_{k}(z)$ and $P_{k-1}(z)$ are polynomials of degree $k$ and $k-1$ respectively, $c_{1} \in \mathbf{C}$. Thus, $R(z)$ has the following form

$$
R(z)=\frac{\prod_{i=1}^{l+n+1}\left(z-\gamma_{i}\right)}{(l+k)(l+k-1) \cdots(l+1)(z-\beta)^{n-k+1}}
$$

where $\gamma_{i}, i=1, \cdots, l+n+1$, and $\beta \in \mathbf{C}$.

Combining Lemma 2.5 and the proof method in [4, Lemma 3.1], we obtain the following result.

Lemma 3.2. Let $k$ be a positive integer, let $\left\{f_{n}\right\}$ be a sequence of meromorphic functions in $D$ and let $\left\{h_{n}\right\}$ be a sequence of meromorphic functions in $D$ such that $h_{n} \stackrel{\chi}{\Longrightarrow} h$ in $D$, where $h \not \equiv 0, \infty$. If

$$
f_{n}(z) \neq 0 \text { and } f_{n}^{(k)}(z) \neq h_{n}(z) \text { for all } z \text { in } D,
$$

then $\mathcal{F}$ is normal in $D$.

Proof. By Lemma 2.5, it suffices to prove that $\left\{f_{n}\right\}$ is normal at points which $h$ has poles or zeros. Without loss of generality, we assume that $D=\Delta, h(z)=z^{l} b(z)$, where $b$ is holomorphic and zero-free in $\Delta, l(\neq 0)$ is an integer. Then $\left\{f_{n}\right\}$ is normal in $\Delta^{\prime}$.

Suppose $\left\{f_{n}\right\}$ is not normal at $z=0$. Since $f_{n} \neq 0$ in $\Delta$, we have that there exists $r>0, \Delta_{2 r} \subset \Delta$, such that $f_{n} \Rightarrow 0$ in $\Delta_{2 r}^{\prime}$. By Argument Principle, for large enough $n$, we have

$$
n\left(r, \frac{1}{f_{n}^{(k)}-h_{n}}\right)-n\left(r, f_{n}^{(k)}-h_{n}\right)=\frac{1}{2 \pi i} \int_{|z|=r} \frac{f_{n}^{(k+1)}-h_{n}^{\prime}}{f_{n}^{(k)}-h_{n}} \mathrm{~d} z=\frac{1}{2 \pi i} \int_{|z|=r} \frac{h^{\prime}}{h} \mathrm{~d} z=l .
$$

Since $f_{n}^{(k)}(z) \neq h_{n}(z)$, thus $-n\left(r, f_{n}^{(k)}-h_{n}\right)=l$. So $l<0$, and $f_{n}$ has poles (otherwise $f_{n} \stackrel{x}{\Rightarrow} \infty$ in $\Delta^{\prime}$ ) which are different from the poles of $h_{n}$. Thus $n\left(r, f_{n}^{(k)}-\right.$ $\left.h_{n}\right)>-l$, this is a contradiction.

\section{Proof of Theorem 1.1}

Proof. Suppose that $f^{(k)}=h$ has at most finitely many zeros, where $k \geq 2$ is an integer. Our goal is to obtain a contradiction in the sequel.

We claim that there exist $t_{n} \rightarrow \infty$ and $\varepsilon_{n} \rightarrow 0$ such that

$$
S\left(\Delta\left(t_{n}, \varepsilon_{n}\right), f\right)=\frac{1}{\pi} \iint_{\left|z-t_{n}\right|<\varepsilon_{n}}\left[f^{\#}(z)\right]^{2} \mathrm{~d} x \mathrm{~d} y \rightarrow \infty .
$$

Otherwise, there would exist $\varepsilon>0$ and $M>0$ such that for all $z_{0} \in \mathbf{C}$, we get

$$
S\left(\Delta\left(z_{0}, \varepsilon\right), f\right)=\frac{1}{\pi} \iint_{\left|z-z_{0}\right|<\varepsilon}\left[f^{\#}(z)\right]^{2} \mathrm{~d} x \mathrm{~d} y<M .
$$

So

$$
S(r, f)=\frac{1}{\pi} \iint_{|z|<r}\left[f^{\#}(z)\right]^{2} \mathrm{~d} x \mathrm{~d} y=O\left(r^{2}\right) .
$$

Then

$$
T(r, f)=\int_{0}^{r} \frac{S(t)}{t} \mathrm{~d} t=O\left(r^{2}\right)
$$


Meanwhile, by Lemma 2.1, $T(r, h)=A r^{2}(1+o(1))$ as $r \rightarrow \infty$, where $A>0$ is a constant. This contradicts the condition that $T(r, h)=o\{T(r, f)\}$. Thus (4.0.1) is correct.

Let $\omega_{1}, \omega_{2}$ be the two fundamental periods of $h(z)$, and $P(0 \in P)$ be a fundamental parallelogram of $h(z)$. Obviously, there exist integers $i_{n}$ and $j_{n}$ satisfying $z_{n}=t_{n}-i_{n} \omega_{1}-j_{n} \omega_{2} \in P$. We can extract a subsequence (still denoted by $\left\{z_{n}\right\}$ ) such that $z_{n} \rightarrow z_{0}$ as $n \rightarrow \infty$. Let $f_{n}(z)=f\left(z+i_{n} \omega_{1}+j_{n} \omega_{2}\right)$. By (4.0.1),

$$
S\left(\Delta\left(z_{n}, \varepsilon_{n}\right), f_{n}\right)=S\left(\Delta\left(t_{n}, \varepsilon_{n}\right), f\right) \rightarrow \infty .
$$

Thus, there exists $z_{n}^{*}\left(z_{n}^{*} \rightarrow z_{0}\right)$ such that $f_{n}^{\#}\left(z_{n}^{*}\right) \rightarrow \infty$ as $n \rightarrow \infty$. Without loss of generality, we suppose that $z_{0}=0$. Hence no subsequence of $\left\{f_{n}\right\}$ is normal at 0 . By (4.0.2),

$$
S\left(\Delta\left(z_{n}^{*}, \varepsilon_{n}^{*}\right), f_{n}\right) \rightarrow \infty,
$$

where $z_{n}^{*} \rightarrow 0$ and $\varepsilon_{n}^{*} \rightarrow 0$ as $n \rightarrow \infty$.

Clearly, there exists $R>0$ such that for all $n, \bar{P} \subset \Delta(0, R)$ and $\Delta\left(z_{n}^{*}, \varepsilon_{n}^{*}\right) \subset$ $\Delta(0, R)$. Let $D=\Delta(0, R)$. Obviously, we have $z_{0} \in D$. By assumption, for large enough $n$,

$$
f_{n}^{(k)}(z)=f^{(k)}\left(z+i_{n} \omega_{1}+j_{n} \omega_{2}\right) \neq h\left(z+i_{n} \omega_{1}+j_{n} \omega_{2}\right)=h(z), \quad z \in D .
$$

Without loss of generality, we suppose that for all $n \in \mathbf{N}, f_{n}^{(k)}(z) \neq h(z)$ in $D$. Obviously, by Lemma 2.9, $\left\{f_{n}\right\}$ is quasinormal in $D$. By (4.0.3), no subsequence of $\left\{f_{n}\right\}$ is normal at 0 . Thus, there exists $\rho>0$, such that $\left\{f_{n}\right\}$ is normal in $\Delta^{\prime}(0, \rho)$, and $h(z) \neq 0, \infty$ in $\Delta^{\prime}(0, \rho)$. Without loss of generality, we may assume that $\rho=1$. Evidently, there exists a subsequence of $\left\{f_{n}\right\}$ (still denoted by $\left\{f_{n}\right\}$ ) such that

(a) all zeros of $\left\{f_{n}\right\}$ are of multiplicity at least $k+1$ in $\Delta$,

(b) for each $n \in \mathbf{N}, f_{n}^{(k)}(z) \neq h(z)$ in $\Delta$, where $h \neq 0, \infty$ in $\Delta^{\prime}$,

(c) no subsequence of $\left\{f_{n}\right\}$ is normal at 0 ,

(d) $f_{n}(z) \Rightarrow f_{0}(z)$ in $\Delta^{\prime}$.

Case 1. $h(0) \neq 0, \infty$. It follows from Lemma 2.8 that there exists $0<\delta<1$ such that for large enough $n, f_{n}$ has a single zero of order $k+1$ in $\Delta(0, \delta)$. By Lemma 2.2, there exists $M>0$ such that $S\left(\frac{\delta}{2}, f_{n}\right)<M$. This contradicts (4.0.3).

Case 2. $h(0)=0$. We assume that 0 is a zero of order $l$ of $h(z)$, where $l$ is a positive integer. Without loss of generality, we may assume that

$$
h(z)=z^{l}+a_{l+1} z^{l+1}+\cdots=z^{l} \widehat{h}(z), \quad z \in \Delta,
$$

where $\widehat{h}(z) \neq 0, \infty$ in $\Delta(0,1)$ and $\widehat{h}(0)=1$.

We claim that for any $\delta>0$, there exists at least one zero of $f_{n}$ in $\Delta(0, \delta)$ for large enough $n$. Otherwise, there exist $\delta_{0}>0$ and a subsequence of $\left\{f_{n}\right\}$ (still denoted by $\left.\left\{f_{n}\right\}\right)$ such that $f_{n}(z) \neq 0$ in $\Delta\left(0, \delta_{0}\right)$. By Lemma 3.2, $\left\{f_{n}\right\}$ is normal at 0 which contradicts (c).

Taking a subsequence and renumbering if necessary, we may assume that $a_{n} \rightarrow 0$ is the zero of $f_{n}$ of smallest modulus. By (a) and (b), we have $f_{n}(0) \neq 0$. Then, $a_{n} \neq 0$. Let $F_{n}(\zeta)=\frac{f_{n}\left(a_{n} \zeta\right)}{a_{n}^{k+l}}$. We have that

(a1) $F_{n}(\zeta) \neq 0$ in $\Delta$,

(a2) all zeros of $F_{n}(\zeta)$ are of multiplicity at least $k+1$,

(a3) $F_{n}^{(k)}(\zeta) \neq \zeta^{l} \widehat{h}\left(a_{n} \zeta\right), F_{n}(1)=0$, and $\zeta^{l} \widehat{h}\left(a_{n} \zeta\right) \stackrel{\chi}{\Longrightarrow} \zeta^{l}$ in C. 
By Lemma 3.2 and Lemma $2.9,\left\{F_{n}(\zeta)\right\}$ is normal in $\Delta$ and quasinormal in C. Hence, there exist $E_{1} \subset \mathbf{C}$ and a subsequence of $\left\{F_{n}(\zeta)\right\}$ (still denoted by $\left.\left\{F_{n}(\zeta)\right\}\right)$ such that

(b1) $E_{1}$ has no accumulation point in $\mathbf{C}$,

(b2) $F_{n}(\zeta) \stackrel{\chi}{\Longrightarrow} F(\zeta)$ in $\mathbf{C} \backslash E_{1}$,

(b3) for each $\zeta_{0} \in E_{1}$, no subsequence of $\left\{F_{n}(\zeta)\right\}$ is normal at $\zeta_{0}$.

Evidently, $E_{1} \cap \triangle=\emptyset$ and all zeros of $F(\zeta)$ are of multiplicity at least $k+1$ in $\mathbf{C} \backslash E_{1}$.

Case 2.1. $E_{1}=\emptyset$. By Lemma 2.6, we have either $F^{(k)}(\zeta) \equiv \zeta^{l}$ or $F^{(k)}(\zeta) \neq \zeta^{l}$ in C. If $F^{(k)}(\zeta) \equiv \zeta^{l}$ in $\mathbf{C}$, then $F^{(k)}(1)=1$ which contradicts $F^{(k)}(1)=0$. If $F^{(k)}(\zeta) \neq \zeta^{l}$ in $\mathbf{C}$, by Lemma 2.11, $F$ must be rational. By Lemma 3.1,

$$
F(\zeta)=\frac{\prod_{i=1}^{l+m+1}\left(\zeta-\alpha_{i}\right)}{(l+k)(l+k-1) \cdots(l+1)(\zeta-\beta)^{m-k+1}},
$$

where $m \geq k$ is an integer, $\beta \in \mathbf{C}$ and $\alpha_{i} \neq 0, \beta(1 \leq i \leq l+m+1)$. Thus, we have

$$
F_{n}(\zeta) \stackrel{\chi}{\Longrightarrow} \frac{\prod_{i=1}^{l+m+1}\left(\zeta-\alpha_{i}\right)}{(l+k)(l+k-1) \cdots(l+1)(\zeta-\beta)^{m-k+1}} \text { in } \mathbf{C} \text {. }
$$

By Hurwitz's Theorem, there exist sequences $\zeta_{n, i} \rightarrow \alpha_{i}$ and $\eta_{n, j} \rightarrow \beta$ (counting multiplicities of zeros and poles, respectively), such that for large enough $n, F_{n}\left(\zeta_{n, i}\right)=0$ and $F_{n}\left(\eta_{n, j}\right)=\infty$, where $i=1,2, \cdots, m+l+1$ and $j=1,2, \cdots, m-k+1$. Let $z_{n, i}=a_{n} \zeta_{n, i}$. Hence, $f_{n}\left(z_{n, i}\right)=0$ and $z_{n, i} \rightarrow 0$ as $n \rightarrow \infty$ where $i=1,2, \cdots, m+l+1$. Let

$$
B_{n}=\left\{z_{n, 1}, z_{n, 2}, \cdots, z_{n, m+l+1}\right\} \quad \text { (where the same elements are admissible). }
$$

Subcase 2.1.1. There exists $\delta(>0)$ and a subsequence of $\left\{f_{n}\right\}$ (still denoted by $\left.\left\{f_{n}\right\}\right)$ such that $f_{n}(z)$ has exactly $m+l+1$ zeros (counting multiplicities) in $\Delta(0, \delta)$ for large enough $n$.

By Lemma 2.2, there exists $M>0$ such that for large enough $n, S\left(\frac{\delta}{2}, f_{n}\right)<M$. This contradicts (4.0.3).

Subcase 2.1.2. For any $\delta>0, f_{n}$ has at least $m+l+2$ zeros (counting multiplicities) in $\Delta(0, \delta)$ for large enough $n$.

Taking a subsequence and renumbering if necessary, we may assume that $b_{n} \neq 0$ is the zero of $f_{n}$ of smallest modulus in $\Delta \backslash B_{n}$. Obviously, we have $b_{n} \rightarrow 0$ as $n \rightarrow \infty$. Let $r_{n}=\frac{a_{n}}{b_{n}}$. Clearly, $F_{n}\left(\frac{1}{r_{n}}\right)=0$. Since $b_{n} \notin B_{n}$, we have $\frac{1}{r_{n}} \neq \zeta_{n, i}$, where $i=1,2, \cdots, m+l+1$. By Hurwitz's Theorem and (4.0.4), $\frac{1}{r_{n}} \rightarrow \infty$, then $r_{n} \rightarrow 0$ as $n \rightarrow \infty$.

Let $G_{n}(\zeta)=\frac{f_{n}\left(b_{n} \zeta\right)}{b_{n}^{k+l}}$. We have that for large enough $n$,

(c1) $G_{n}(\zeta)$ has only $m+l+1$ zeros $r_{n} \zeta_{n, i}$ in $\Delta$ and $\left|r_{n} \zeta_{n, i}\right| \rightarrow 0$, as $n \rightarrow \infty$, where $i=1,2, \cdots, m+l+1$,

(c2) all zeros of $G_{n}(\zeta)$ are of multiplicity at least $k+1$,

(c3) $G_{n}^{(k)}(\zeta) \neq \zeta^{l} \widehat{h}\left(b_{n} \zeta\right)$ and $G_{n}(1)=0$.

By Lemma 3.2 and Lemma 2.9, $\left\{G_{n}(\zeta)\right\}$ is normal in $\Delta^{\prime}$ and quasinormal in $\mathbf{C}$. Hence, there exist $E_{2} \subset \mathbf{C}$ and a subsequence of $\left\{G_{n}(\zeta)\right\}$ (still denoted by $\left\{G_{n}(\zeta)\right\}$ ) such that 
(d1) $E_{2}$ has no accumulation point in $\mathbf{C}$,

(d2) $G_{n}(\zeta) \stackrel{\chi}{\Longrightarrow} G(\zeta)$ in $\mathbf{C} \backslash E_{2}$,

(d3) for each $\zeta_{0} \in E_{2}$, no subsequence of $\left\{G_{n}(\zeta)\right\}$ is normal at $\zeta_{0}$.

Evidently, $E_{2} \cap \Delta^{\prime}=\emptyset$ and all zeros of $G(\zeta)$ are of multiplicity at least $k+1$ in $\mathrm{C} \backslash E_{2}$.

Let

$$
G_{n}^{*}(\zeta)=G_{n}(\zeta) \frac{\prod_{j=1}^{m-k+1}\left(\zeta-r_{n} \eta_{n, j}\right)}{\prod_{i=1}^{m+l+1}\left(\zeta-r_{n} \zeta_{n, i}\right)}, \quad F_{n}^{*}(\zeta)=F_{n}(\zeta) \frac{\prod_{j=1}^{m-k+1}\left(\zeta-\eta_{n, j}\right)}{\prod_{i=1}^{m+l+1}\left(\zeta-\zeta_{n, i}\right)} .
$$

By $(4.0 .4), G_{n}^{*}\left(r_{n} \zeta\right)=F_{n}^{*}(\zeta) \Rightarrow \frac{1}{(l+k)(l+k-1) \cdots(l+1)}$ in C. Thus

$$
G_{n}^{*}(0) \rightarrow G^{* *}(0)=\frac{1}{(l+k)(l+k-1) \cdots(l+1)} .
$$

Subcase 2.1.2.1. $G(\zeta) \equiv \infty$ in $\mathbf{C} \backslash E_{2}$. Evidently, $G_{n}^{*}(\zeta)$ has no zeros in $\Delta$. We have $G_{n}^{*}(\zeta) \Rightarrow \infty$ in $\Delta$ which follows from Maximum Modulus Principle of analytic functions. This contradicts (4.0.5).

Subcase 2.1.2.2. $G(\zeta)$ is a meromorphic function in $\mathbf{C} \backslash E_{2}$. We claim that

$$
G(\zeta)=\frac{\zeta^{l+k}}{(l+k)(l+k-1) \cdots(l+1)} \text { in } \mathbf{C} \backslash E_{2} .
$$

By Lemma 2.7, $G^{(k)}(\zeta) \equiv \zeta^{l}$, then $G(\zeta)=\frac{\zeta^{l+k}+P_{k-1}(\zeta)}{(l+k)(l+k-1) \cdots(l+1)}$, where $P_{k-1}(z)$ is a polynomial of degree $\leq k-1$. Since $G_{n}^{*}(\zeta)$ have no zeros in $\Delta$,

$$
G_{n}^{*}(\zeta) \Rightarrow G^{*}(\zeta)=\frac{\zeta^{l+k}+P_{k-1}(\zeta)}{(l+k)(l+k-1) \cdots(l+1)} \frac{1}{\zeta^{l+k}}, \quad \zeta \in \Delta .
$$

On one hand, by (4.0.5), we have $G^{* *}(0) \neq 0, \infty$. And by (4.0.5) and (4.0.7), we obtain $G^{* *}(0)=G^{*}(0)$. Then we have $G^{*}(0) \neq 0, \infty$. On the other hand, by the expression of $G^{*}(\zeta)$ in (4.0.7), 0 is the zero of the denominator of multiplicity $l+k$. Then, 0 is the zero of the numerator of multiplicity $l+k$. Hence, 0 is the zero of $P_{k-1}(\zeta)$ of multiplicity $l+k$. However, $P_{k-1}(z)$ is a polynomial of degree $\leq k-1$. Thus, we have $P_{k-1}(\zeta) \equiv 0$.

If $1 \notin E_{2}$, by (c3), we have $G(1)=0$. This contradicts (4.0.6). If $1 \in E_{2}$, by Lemma 2.8, $G^{(k-1)}(\zeta)=\int_{1}^{\zeta} \xi^{l} \mathrm{~d} \xi=\frac{\zeta^{l+1}-1}{l+1}$. However, by (4.0.6), we have $G^{(k-1)}(\zeta)=$ $\frac{\zeta^{l+1}}{l+1}$. This is a contradiction.

Subcase 2.2. $E_{1} \neq \emptyset$. We claim that $1 \in E_{1}$. Suppose that $1 \notin E_{1}$. Since $E_{1} \neq \emptyset$, there exists at least a point $\zeta_{0}$ such that $\zeta_{0} \in E_{1}$. Thus, by Lemma $2.8, F^{(k)}(\zeta)=\zeta^{l}$ in $\mathbf{C} \backslash E_{1}$. At the same time, $F(1)=0$ and all zeros of $F$ are of multiplicity at least $k+1$, we have $F^{(k)}(1)=0$, a contradiction.

Thus, by Lemma 2.8, in $\mathbf{C} \backslash E_{1}$,

$$
\begin{aligned}
& F^{(k-1)}(\zeta)=\int_{1}^{\zeta} \xi^{l} \mathrm{~d} \xi=\frac{\zeta^{l+1}-1}{l+1} . \\
& F^{(k-2)}(\zeta)=\int_{1}^{\zeta} \frac{\xi^{l+1}-1}{l+1} \mathrm{~d} \xi=\frac{\zeta^{l+2}}{(l+2)(l+1)}-\frac{\zeta}{l+1}+\frac{1}{(l+2)} .
\end{aligned}
$$


We claim that $E_{1}=\{1\}$. Otherwise, there exists $\zeta_{0} \in E_{1}$ and $\zeta_{0} \neq 1$. By Lemma 2.8, in $\mathbf{C} \backslash E_{1}$,

$$
F^{(k-1)}(\zeta)=\int_{\zeta_{0}}^{\zeta} \xi^{l} \mathrm{~d} \xi=\frac{\zeta^{l+1}-\zeta_{0}^{l+1}}{l+1} .
$$

By (4.0.8) and (4.0.10), we obtain $\zeta_{0}^{l+1}=1$. Furthermore, by Lemma 2.8, in $\mathbf{C} \backslash E_{1}$,

$$
F^{(k-2)}(\zeta)=\int_{\zeta_{0}}^{\zeta} \frac{\xi^{l+1}-1}{l+1} \mathrm{~d} \xi=\frac{\zeta^{l+2}}{(l+2)(l+1)}-\frac{\zeta}{l+1}+\frac{\zeta_{0}}{(l+2)} .
$$

By (4.0.9) and (4.0.11), we get $\zeta_{0}=1$, a contradiction. Thus $E_{1}=\{1\}$.

By Lemma 2.8, $F(z)$ can extend to an analytic function in $\mathbf{C}$ and is a polynomial of degree $l+k$. 1 is the zero of $F$ with multiplicity $k$. Hence, $F$ must have zeros which are distinct from 1 . We may denote one of the other zeros of $F$ by $e_{1}$. Since all zeros of $F(\zeta)$ are of multiplicity at least $k+1$ in $\mathbf{C} \backslash E_{1}$, we have $F^{(k-1)}\left(e_{1}\right)=0, F^{(k)}\left(e_{1}\right)=0$. Then, $e_{1}$ is a multiple zero of $F^{(k-1)}(z)$. However, by $(4.0 .8), F^{(k-1)}(z)$ only has simple roots. This is a contradiction.

Case 3. $h(0)=\infty$. Suppose that $z=0$ is a pole of order $l$ of $h(z)$. Without loss of generality, we may assume that

$$
h(z)=\frac{1}{z^{l}}+\frac{a_{l-1}}{z^{l-1}}+\cdots=\frac{\widehat{h}(z)}{z^{l}} \text { in } \Delta,
$$

where $\widehat{h}(z) \neq 0, \infty$ in $\Delta$ and $\widehat{h}(0)=1$.

Subcase 3.1. For $n$ sufficiently large, $f_{n}(0) \neq 0$. Without loss of generality, we assume that for each $n \in \mathbf{N}, f_{n}(0) \neq 0$.

We claim that for any $\delta>0$, there exists at least one zero of $f_{n}$ in $\Delta(0, \delta)$ for sufficiently large $n$. Otherwise, there exist $\delta_{0}(>0)$ and a subsequence of $\left\{f_{n}\right\}$ (still denoted by $\left.\left\{f_{n}\right\}\right)$ such that $f_{n}(z) \neq 0$ in $\Delta\left(0, \delta_{0}\right)$. By Lemma $3.2,\left\{f_{n}\right\}$ is normal at 0 . This is a contradiction.

Taking a subsequence and renumbering if necessary, we may assume that $a_{n}$ is the zero of $\left\{f_{n}\right\}$ of smallest modulus. Evidently, $a_{n} \rightarrow 0$ as $n \rightarrow \infty$. Since $f_{n}(0) \neq 0$, we have $a_{n} \neq 0$.

Let $F_{n}(\zeta)=a_{n}^{l-k} f_{n}\left(a_{n} \zeta\right)$, clearly we have

(i1) $F_{n}(\zeta) \neq 0$ in $\Delta$,

(i2) all zeros of $F_{n}(\zeta)$ are of multiplicity at least $k+1$,

(i3) $F_{n}^{(k)}(\zeta) \neq \frac{\widehat{h}\left(a_{n} \zeta\right)}{\zeta^{l}}, F_{n}(1)=0$, and $\frac{\widehat{h}\left(a_{n} \zeta\right)}{\zeta^{l}} \stackrel{\chi}{\Longrightarrow} \frac{1}{\zeta^{l}}$ in $\mathbf{C}$.

By Lemma 3.2 and Lemma 2.9, $\left\{F_{n}(\zeta)\right\}$ is normal in $\Delta$ and quasinormal in C. Obviously, there exist $E_{3} \subset \mathbf{C}$ and a subsequence of $\left\{F_{n}(\zeta)\right\}$ (still denoted by $\left\{F_{n}(\zeta)\right\}$ ) such that

(j1) $E_{3}$ has no accumulation point in $\mathbf{C}$,

(j2) $F_{n}(\zeta) \stackrel{\chi}{\Longrightarrow} F(\zeta)$ in $\mathbf{C} \backslash E_{3}$,

(j3) for each $\zeta_{0} \in E_{3}$, no subsequence of $\left\{F_{n}(\zeta)\right\}$ is normal at $\zeta_{0}$.

Clearly, $E_{3} \cap \Delta=\emptyset$ and all zeros of $F(\zeta)$ are of multiplicity at least $k+1$ in $\mathbf{C} \backslash E_{3}$.

Subcase 3.1.1. $\quad E_{3}=\emptyset$. By (i2), (i3), we have $F(1)=\cdots=F^{(k)}(1)=0$. Thus, $F(\zeta)$ is a meromorphic function in C. Combining (i3) and Lemma 2.6, we have either $F^{(k)}(\zeta) \equiv \frac{1}{\zeta^{l}}$ or $F^{(k)}(\zeta) \neq \frac{1}{\zeta^{l}}$ in $\mathbf{C}$.

If $F^{(k)}(\zeta) \equiv \frac{1}{\zeta^{l}}$ in $\mathbf{C}$, which contradicts $F^{(k)}(1)=0$. 
If $F^{(k)}(\zeta) \neq \frac{1}{\zeta^{l}}$ in $\mathbf{C}$, by Lemma 2.11 and Lemma 2.3, $F(\zeta)=c$. Since $F(1)=0$, we have $F(\zeta) \equiv 0$, i.e.,

$$
F_{n}(\zeta)=a_{n}^{l-k} f_{n}\left(a_{n} \zeta\right) \Rightarrow 0 \text { in } \mathbf{C}
$$

We claim that for any $\delta>0$, there exists at least one pole of $f_{n}$ in $\Delta(0, \delta)$ for sufficiently large $n$. Otherwise, there exist $\delta_{0}(>0)$ and a subsequence of $\left\{f_{n}\right\}$ (still denoted by $\left.\left\{f_{n}\right\}\right)$ such that $\left\{f_{n}\right\}$ is of a family of holomorphic in $\Delta\left(0, \delta_{0}\right)$. By Lemma 2.10, $\left\{f_{n}\right\}$ is normal at 0 . This is a contradiction.

Taking a subsequence and renumbering if necessary, we may assume that $b_{n}$ is the pole of $f_{n}(z)$ of smallest modulus. Clearly, we have $b_{n} \rightarrow 0$ as $n \rightarrow \infty$. Since $f_{n}^{(k)}(z) \neq h(z)$ and $h(0)=\infty$, we have $f(0) \neq \infty$, hence $b_{n} \neq 0$.

Let $G_{n}(\zeta)=b_{n}^{l-k} f_{n}\left(b_{n} \zeta\right)$. Clearly, we have

(k1) $G_{n}(\zeta)$ is holomorphic function in $\Delta$,

(k2) all zeros of $G_{n}(\zeta)$ are of multiplicity at least $k+1$,

(k3) $G_{n}^{(k)}(\zeta) \neq \frac{\widehat{h}\left(b_{n} \zeta\right)}{\zeta^{l}}, G_{n}(1)=\infty$ and $\frac{\widehat{h}\left(b_{n} \zeta\right)}{\zeta^{l}} \stackrel{\chi}{\Longrightarrow} \frac{1}{\zeta^{l}}$ in C.

By Lemma 2.10 and Lemma 2.9, $\left\{G_{n}(\zeta)\right\}$ is normal in $\Delta$ and quasinormal in C. Thus, there exist $E_{4} \subset \mathbf{C}$ and a subsequence of $\left\{G_{n}(\zeta)\right\}$ (still denoted by $\left\{G_{n}(\zeta)\right\}$ ) such that

(11) $E_{4}$ has no accumulation point in $\mathbf{C}$,

(12) $G_{n}(\zeta) \stackrel{\chi}{\Longrightarrow} G(\zeta)$ in $\mathbf{C} \backslash E_{4}$,

(13) for each $\zeta_{0} \in E_{4}$, no subsequence of $\left\{G_{n}(\zeta)\right\}$ is normal at $\zeta_{0}$.

Evidently, $E_{4} \cap \Delta=\emptyset$ and all zeros of $G(\zeta)$ are of multiplicity at least $k+1$ in $\mathbf{C} \backslash E_{4}$.

Since $F_{n}\left(\frac{b_{n}}{a_{n}}\right)=a_{n}^{l-k} f_{n}\left(b_{n}\right)=\infty$, by Hurwitz's theorem and (4.0.12), $\frac{b_{n}}{a_{n}} \rightarrow \infty$. Set $r_{n}=\frac{a_{n}}{b_{n}}$. Evidently, $r_{n} \rightarrow 0$ as $n \rightarrow \infty$. Since $G_{n}\left(r_{n}\right)=b_{n}^{l-k} f_{n}\left(a_{n}\right)=0$, we have $G(0)=0$. Hence $G(z)$ is a meromorphic function in $\mathbf{C} \backslash E_{4}$. By (k3) and Lemma 2.6, we have either $G^{(k)}(\zeta) \equiv \frac{1}{\zeta^{l}}$ or $G^{(k)}(\zeta) \neq \frac{1}{\zeta^{l}}$ in $\mathbf{C} \backslash E_{4}$.

We now consider two subcases.

Subcase 3.1.1.1. $E_{4}$ is an empty set. If $G^{(k)}(\zeta) \equiv \frac{1}{\zeta^{l}}$ in $\mathbf{C}$, we have $G(0)=\infty$ which contradicts $G(0)=0$. If $G^{(k)}(\zeta) \neq \frac{1}{\zeta^{l}}$ in $\mathbf{C}$, by Lemma 2.11 and Lemma 2.3, $G(\zeta)=c$ which contradicts $G(1)=\infty$.

Subcase 3.1.1.2. $E_{4}$ is not an empty set. Let $\zeta_{0} \in E_{4}$. Clearly, $\zeta_{0} \neq 0$. By Lemma 2.8, $G^{(k-1)}(\zeta)=\int_{\zeta_{0}}^{\zeta} \frac{1}{\xi^{l}} \mathrm{~d} \xi=\frac{1}{1-l}\left(\frac{1}{\zeta^{l-1}}-\frac{1}{\zeta_{0}{ }^{l-1}}\right)$. Evidently, $G(0)=\infty$ which contradicts $G(0)=0$.

Subcase 3.1.2. $E_{3} \neq \emptyset$. We claim that $1 \in E_{3}$. Suppose that $1 \notin E_{3}$. Since $E_{3} \neq \emptyset$, there exists at least a point $\zeta_{0}$ such that $\zeta_{0} \in E_{3}$. Thus, by Lemma 2.8, $F^{(k)}(\zeta)=\frac{1}{\zeta^{l}}$ in $\mathbf{C} \backslash E_{3}$. Meanwhile, $F(1)=0$ and all zeros of $F$ are of multiplicity at least $k+1$, we have $F^{(k)}(1)=0$, a contradiction.

Thus, by Lemma 2.8, in $\mathbf{C} \backslash E_{3}$,

$$
\begin{aligned}
& F^{(k-1)}(\zeta)=\int_{1}^{\zeta} \frac{1}{\xi^{l}} \mathrm{~d} \xi=\frac{\zeta^{-l+1}-1}{-l+1} \\
& F^{(k-2)}(\zeta)=\int_{1}^{\zeta} \frac{\xi^{-l+1}-1}{-l+1} \mathrm{~d} \xi=\frac{\zeta^{-l+2}}{(-l+2)(-l+1)}-\frac{\zeta}{-l+1}+\frac{1}{(-l+2)}
\end{aligned}
$$


We claim that $E_{3}=\{1\}$. Otherwise, there exists $\zeta_{0} \in E_{1}$ and $\zeta_{0} \neq 1$. By Lemma 2.8, in $\mathbf{C} \backslash E_{3}$,

$$
F^{(k-1)}(\zeta)=\int_{\zeta_{0}}^{\zeta} \frac{1}{\xi l} \mathrm{~d} \xi=\frac{\zeta^{-l+1}-\zeta_{0}^{-l+1}}{-l+1} .
$$

By (4.0.13) and (4.0.15), we obtain $\zeta_{0}^{-l+1}=1$. Furthermore, by Lemma 2.8, in $\mathrm{C} \backslash E_{3}$,

$$
F^{(k-2)}(\zeta)=\int_{\zeta_{0}}^{\zeta} \frac{\xi^{-l+1}-1}{-l+1} \mathrm{~d} \xi=\frac{\zeta^{-l+2}}{(-l+2)(-l+1)}-\frac{\zeta}{-l+1}+\frac{\zeta_{0}}{(l+2)} .
$$

By (4.0.14) and (4.0.16), we get $\zeta_{0}=1$, a contradiction. Thus $E_{3}=\{1\}$.

Thus, by Lemma 2.8, in $\mathbf{C} \backslash E_{3}$

$$
\begin{aligned}
F(\zeta) & =\int_{\zeta=1}^{\zeta} \int_{\zeta=1}^{\zeta_{1}} \cdots \int_{\zeta=1}^{\zeta_{k-1}} \frac{1}{\zeta_{k}^{l}} \mathrm{~d} \zeta_{k} \mathrm{~d} \zeta_{k-1} \cdots \mathrm{d} \zeta_{1} \\
& =\frac{1+P_{k-1}(\zeta) \zeta^{l-k}}{(-l+k)(-l+k-1) \cdots(-l+1) \zeta^{l-k}},
\end{aligned}
$$

where $P_{k-1}(\zeta)$ is a polynomial of degree $k-1$. Hence, by (4.0.17), in $\Delta$

$$
F_{n}(\zeta) \stackrel{\chi}{\Longrightarrow} \frac{1+P_{k-1}(\zeta) \zeta^{l-k}}{(-l+k)(-l+k-1) \cdots(-l+1) \zeta^{l-k}} .
$$

By Hurwitz's Theorem, there exist $\left.\gamma_{n, i}, i=1,2, \ldots, l-k\right)$, such that $\gamma_{n, i} \rightarrow 0$ and $F_{n}\left(\gamma_{n, i}\right)=\infty$. Since $f$ and $h$ have no common poles, we have $F_{n}(0) \neq \infty, \gamma_{n, i} \neq 0$, $i=1,2, \cdots, l-k$.

Suppose that $s_{n}$ is one of $\left\{\gamma_{n, 1}, \gamma_{n, 2}, \cdots, \gamma_{n, l-k}\right\}$ of largest modulus. Let $U_{n}(\xi)=$ $s_{n}^{l-k} F_{n}\left(s_{n} \xi\right)$. Evidently, $U_{n}(\xi)$ has only $l-k$ poles $\eta_{n, i}=\frac{\gamma_{n, i}}{s_{n}}$ on $\bar{\Delta}$ for $n$ sufficiently large. By the expression of $F^{(k-1)}$ in (4.0.13), for all $R>0$ and large enough $n$,

$$
U_{n}(\xi) \neq 0, \quad \xi \in \Delta(0, R) .
$$

Combining (i3) and $U_{n}^{(k)}(\xi) \neq \frac{\widehat{h}\left(a_{n} s_{n} \xi\right)}{\xi^{l}}$, and by Lemma 3.2, $U_{n}(\xi)$ is normal in C. We assume that $U_{n}(\xi) \stackrel{\chi}{\Longrightarrow} U(\xi)$ in $\mathbf{C}$ and $\eta_{n, i} \rightarrow \eta_{i}$. Since $U_{n}(1)=\infty$, we have $U(1)=\infty$.

We claim that $U(\xi) \equiv \infty$ in C. Otherwise, by Lemma 2.6, we have either $U^{(k)}(\zeta) \equiv \frac{1}{\xi^{l}}$ or $U^{(k)}(\xi) \neq \frac{1}{\xi^{l}}$ in C. If $U^{(k)}(\xi) \equiv \frac{1}{\xi^{l}}$ in $\mathbf{C}$, which contradicts $U(1)=\infty$. If $U^{(k)}(\xi) \neq \frac{1}{\xi^{l}}$ in $\mathbf{C}$, by Lemma 2.11 and Lemma 2.3, we have $U(\xi)=c$, where $c$ is a constant. This contradicts $U(1)=\infty$. Thus, $U_{n}(\xi) \stackrel{\chi}{\Longrightarrow} \infty$ in C.

Let

$$
U_{n}^{*}(\xi)=U_{n}(\xi) \cdot \prod_{i=1}^{l-k}\left(\xi-\eta_{n, i}\right)
$$

Furthermore, we obtain $U_{n}^{*}(\xi) \stackrel{\chi}{\Longrightarrow} \infty$ in C.

Let

$$
F_{n}^{*}(\zeta)=F_{n}(\zeta) \cdot \prod_{i=1}^{l-k}\left(\zeta-\gamma_{n, i}\right)=F_{n}(\zeta) \cdot \prod_{i=1}^{l-k}\left(\zeta-s_{n} \eta_{n, i}\right)
$$


By $(4.0 .18), F_{n}^{*}(\zeta)$ has no poles in $\Delta\left(0, \frac{1}{2}\right)$ for large enough $n$. And by the maximum principle,

$$
F_{n}^{*}(\zeta) \stackrel{\chi}{\Longrightarrow} \frac{1+P_{k-1}(\zeta) \zeta^{l-k}}{(-l+k)(-l+k-1) \cdots(-l+1)} \quad \text { in } \Delta\left(0, \frac{1}{2}\right)
$$

Hence,

$$
F_{n}^{*}(0) \rightarrow \frac{1}{(-l+k)(-l+k-1) \cdots(-l+1)} \quad \text { as } n \rightarrow \infty .
$$

On the other hand, in $\mathbf{C}$

$$
\begin{aligned}
F_{n}^{*}\left(s_{n} \xi\right) & =F_{n}\left(s_{n} \xi\right) \cdot \prod_{i=1}^{l-k}\left(s_{n} \xi-\gamma_{n, i}\right)=F_{n}\left(s_{n} \xi\right) \cdot \prod_{i=1}^{l-k}\left(s_{n} \xi-s_{n} \eta_{n, i}\right) \\
& =s_{n}^{l-k} F_{n}\left(s_{n} \xi\right) \cdot \prod_{i=1}^{l-k}\left(\xi-\eta_{n, i}\right)=U_{n}^{*}(\xi) \stackrel{\chi}{\Longrightarrow} \infty
\end{aligned}
$$

By (4.0.20), $F_{n}^{*}(0) \rightarrow \infty$ as $n \rightarrow \infty$ which contradicts (4.0.19).

Subcase 3.2. There exists a subsequence of $\left\{f_{n}(z)\right\}$ (still denoted by $\left\{f_{n}(z)\right\}$ ) such that $f_{n}(0)=0$. We may assume that $c_{n}(\neq 0)$ is the pole of $f_{n}(z)$ of smallest modulus and $c_{n} \rightarrow 0$ as $n \rightarrow \infty$ which is similar to Subcase 3.1.1..

Let $G_{n}(\zeta)=c_{n}^{l-k} f_{n}\left(c_{n} \zeta\right)$. Obviously, we have

(m1) $G_{n}(\zeta)$ is holomorphic function in $\Delta$,

(m2) all zeros of $G_{n}(\zeta)$ are of multiplicity at least $k+1$,

(m3) $G_{n}^{(k)}(\zeta) \neq \frac{\widehat{h}\left(c_{n} \zeta\right)}{\zeta^{l}}, G_{n}(1)=\infty$, and $\frac{\widehat{h}\left(c_{n} \zeta\right)}{\zeta^{l}} \stackrel{\chi}{\Longrightarrow} \frac{1}{\zeta^{l}}$ in $\mathbf{C}$.

By Lemma 2.10 and Lemma 2.9, $\left\{G_{n}(\zeta)\right\}$ is normal in $\Delta$ and quasinormal in C. Then, there exist $E_{5} \subset \mathbf{C}$ and a subsequence of $\left\{G_{n}(\zeta)\right\}$ (still denoted by $\left\{G_{n}(\zeta)\right\}$ ) satisfying

(n1) $E_{5}$ has no accumulation point in $\mathbf{C}$,

(n2) $G_{n}(\zeta) \stackrel{\chi}{\Longrightarrow} G(\zeta)$ in $\mathbf{C} \backslash E_{5}$,

(n3) for each $\zeta_{0} \in E_{5}$, no subsequence of $\left\{G_{n}(\zeta)\right\}$ is normal at $\zeta_{0}$.

Evidently, $E_{5} \cap \Delta=\emptyset$ and all zeros of $G(\zeta)$ are of multiplicity at least $k+1$ in $\mathbf{C} \backslash E_{5}$.

Clearly, $G_{n}(0)=c_{n}^{l-k} f_{n}(0)=0$, we have $G(0)=0$. Hence, $G(z)$ is meromorphic in $\mathbf{C} \backslash E_{5}$. By (m3) and Lemma 2.6, we have either $G^{(k)}(\zeta) \equiv \frac{1}{\zeta^{l}}$ or $G^{(k)}(\zeta) \neq \frac{1}{\zeta^{l}}$ in $\mathrm{C} \backslash E_{5}$.

We now distinguish two cases.

Case 3.2.1. $E_{5}$ is an empty set. If $G^{(k)}(\zeta) \equiv \frac{1}{\zeta^{2}}$ in $\mathbf{C}$, we have $G(0)=\infty$ which contradicts $G(0)=0$. If $G^{(k)}(\zeta) \neq \frac{1}{\zeta^{l}}$ in $\mathbf{C}$, by Lemma 2.11 and Lemma 2.3, $G(\zeta)=c$ which contradicts $G(1)=\infty$.

Case 3.2.2. $E_{5}$ is not an empty set. Let $\zeta_{0} \in E_{5}$. Clearly, we have $\zeta_{0} \neq 0$. By Lemma 2.8, we get $G^{(k-1)}(\zeta)=\int_{\zeta_{0}}^{\zeta} \frac{1}{\xi^{l}} \mathrm{~d} \xi=\frac{1}{1-l}\left(\frac{1}{\zeta^{l-1}}-\frac{1}{\zeta_{0}{ }^{l-1}}\right)$. Evidently, we have $G(0)=\infty$ which contradicts $G(0)=0$.

Acknowledgements. We are very grateful to the reviewers for their valuable suggestions. 


\section{References}

[1] Akhiezer, N. I.: Elements of the theory of elliptic functions. - Transl. Math. Monogr. 79, Amer. Math. Soc., Rhode Island, 1990.

[2] Bank, S.B., and J.K. LAngley: On the value distribution theory of elliptic functions. Monatsh. Math. 98, 1984, 1-20.

[3] Chang, J. M., and L. Zalcman: Meromorphic functions that share a set with their derivatives. - J. Math. Anal. Appl. 338, 2008, 1020-1028.

[4] Chen, Q. Y., YAng, L, and X. C. PAng: Normal family and the sequence of omitted functions. - Sci. China Math. 56, 2013, 1821-1830.

[5] Hayman, W. K.: Picard values of meromorphic functions and their derivatives. - Ann. of Math. (2) 70, 1959, 9-42.

[6] Nevo, S., X. C. PAng, and L. Zalcman: Quasinormality and meromorphic functions with multiple zeros. - J. Anal. Math. 101, 2007, 1-23.

[7] Pang, X. C., S. Nevo, and L. Zalcman: Derivatives of meromorphic functions with multiple zeros and rational functions. - Comput. Methods Funct. Theory 8, 2008, 483-491.

[8] Wang, Y.F., and M. L. FAng: Picard values and normal families of meromorphic functions with multiple zeros. - Acta Math. Sinica (N. S.) 14, 1998, 17-26.

[9] Xu, Y.: Normal families and exceptional functions. - J. Math. Anal. Appl. 329, 2007, 13431354.

[10] YANG, P., and X. J. LIU: On the $k$ th derivatives of meromorphic functions and rational functions. - J. East China Norm. Univ. Natur. Sci. Ed. Jul. 4, 2014, 8-17.

[11] YAnG, P., and S. Nevo: Derivatives of meromorphic functions with multiple zeros and elliptic functions. - Acta Math. Sin. (Engl. Ser.) 29, 2013, 1257-1278.

[12] Zhang, G. M., X. C. Pang, and L. Zalcman: Normal families and omitted functions II. Bull. London Math. Soc. 41, 2009, 63-71.

Received 18 September 2013 • First revised received 25 December $2014 \bullet$ Second revised received 29 April 2014 • Accepted 9 May 2014 